\title{
Performance Evaluation of Mobility Management Scheme in DTN
}

\author{
Mooi Choo Chuah, Vinay Goel, Brian D. Davison \\ Department of Computer Science and Engineering \\ Lehigh University \\ Bethlehem, PA 18015 USA \\ \{chuah, vig204, davison\}@cse.lehigh.edu
}

\begin{abstract}
Standard ad hoc routing protocols do not work in intermittently connected networks since end-to-end paths may not exist in such networks. A store-and-forward approach [7] has been proposed for such networks. The nodes in such networks move around. Thus, the proposed delay tolerant network (DTN) architecture [7] needs to be enhanced with a mobility management scheme to ensure that nodes that wish to correspond with mobile hosts have a way of determining their whereabouts. The mobile hosts may move a short distance and hence remain within the vicinity of a DTN name registrar (DNR) (one communication link away) or they may move far away (multiple communication links away). In this paper, we present the mobility management scheme we propose for DTN environments. In addition, we provide simple analytical formulae to evaluate the latency required for performing location updates, and the useful utilization that each node can use for data transfer assuming that the communication links between nodes are periodically available for a short period of time. Our simple analytical model allows us to draw insights into the impact of near/far movements on the useful utilization.
\end{abstract}

Keywords-delay/disruption tolerant networking, mobility management, EDIFY

\section{INTRODUCTION}

There are many real mobile networks where the wireless devices are intermittently connected. Most of the time, there does not exist a complete path from a source to a destination or such a path is highly unstable and may change after it has been discovered. Examples of such networks are wildlife tracking sensor networks [1], military networks [2], and inter-planetary networks [3].

Since in such intermittently connected networks, there may not exist any end-to-end path between a source and a destination, conventional mobile adhoc network routing protocols such as DSR [4], AODV [5], etc., will not work in such networks. Reactive routing schemes will fail to discover a complete path and proactive routing schemes will fail to converge resulting in spurious topology update

This work is sponsored by Defense Advanced Research Projects Agency (DARPA). Any opinions, findings, and conclusions or recommendations expressed in this material are those of the authors and do not necessarily reflect the views of DARPA. This document is approved for public release, unlimited distribution. messages. However, this does not mean that messages cannot be delivered from the source to the destination. It just means that a message needs to be sent over an existing link, get buffered at the next hop until the next link is up, and so on, until it reaches its destination.

Some researchers working on Delay Tolerant Networks [7] proposed a bundle delivery protocol to allow nodes in intermittently connected networks to communicate via the store-and-forward approach. We have proposed enhancements to the Delay Tolerant Network architecture work which we refer to as EDIFY [6] to deal with mobility. We denote intermittently connected networks that have been enhanced with the bundle delivery protocol and other EDIFY features as disruption tolerant networks (DTNs).

In this paper, we first discuss why the existing mobility management schemes do not work in DTN environments. Then, we provide more details on how mobility management works in EDIFY. The connectivity between two nodes in an intermittently connected network may toggle between on/off states at any time. Thus, it is important to understand what fraction of the on-times a mobile host can use for data transfer after it spends some time performing location updates when it roams around. We refer to this metric as the useful utilization. In this paper, we provide a simple analytical model that allows us to draw insights about the impact of near/far movement on the useful utilization a mobile node can have for data transfer after performing location updates.

The rest of the paper is organized as follows: In Section II, we discuss related work in the area of mobility management and explain why they do not apply to DTN environment. Then, in Section III, we briefly describe the EDIFY architecture. In Section IV, we discuss how mobility management is done in EDIFY. In Section V, we describe an analytical model that allows us to compute the location update latency and useful utilization assuming the links between nodes are available periodically for a certain percentage of the time. Then, via simulations, we show that the results obtained via analysis are confirmed by the simulation results. 


\section{RELATED WORK}

Several network layer mobility solutions, e.g., Mobile IP [11] and SIP mobility [12], have been proposed to provide mobility transparency. However, such protocols cannot work in partitioned networks since the protocols assume that an end-to-end connection exists.

Transport layer mobility solutions also exist. In TCP Migrate [9], the mobile host periodically updates a unique fully-qualified domain name for itself in the Domain Name System (DNS). The corresponding host uses this information to find the current IP address of the mobile. The protocol stacks of both the mobile host and the corresponding host are also modified to migrate the TCP sessions across prolonged disconnections and IP address changes. However, end-to-end connections are still required with this approach. In addition, frequent DNS updates may result in high control overhead.

In his work on Delay Tolerant Networks [7], Kevin Fall proposed a notion of bundle transfer, in which a message is wrapped into bundles and these bundles are delivered from one hop to another, then stored at that next hop before another opportunistic link appears to further the bundles towards their final destinations. Custody transfer occurs as the bundles are transmitted from one hop to another and the responsibility of reliable delivery of each bundle lies in each DTN router that is involved in the delivery path. Note that there is a delivery path but no existing end-to-end path. However, this solution does not support mobility. We have proposed extensions [6] to his approach to deal with mobility. In [10], the authors also propose an architecture to deal with disconnected networks. They propose a cellular-like solution that consists of Home Location Register and Visiting Location Register. The VLR contains information on the custodian DTN router for a visiting mobile.

\section{EDIFY ARCHITECTURE}

In the EDIFY architecture [6], there are several types of nodes, namely (a) regular DTN nodes, (b) DTN Name Registrars (DNRs), and (c) DTN gateways. We briefly describe the functionalities provided by each type of nodes below:

- Regular DTN nodes. In EDIFY, all nodes participating in the DTN have the ability to send and receive bundles to other nearby nodes using the underlying networking infrastructure. When a node joins a group, the node is informed of a default node (a gateway) to which bundles may be sent. The node also implicitly knows of the group registrar, from which each node acquires its name within the group. Every node in the DTN can have one or more names but only one identifier will be selected to be their "home name", e.g., bob@cse.lehigh.edu.
- DTN gateways. A DTN gateway is a DTN node that offers forwarding services to one or more destination groups. Nodes that perform forwarding services form the backbone of the DTN. DTN gateways may also advertise the availability of routing services to non-local groups to other gateways inside or outside of the group to which a gateway belongs.

- DTN Name Registrar (DNR). Every group in the DTN world has a registrar (DNR) associated with it. We assume that the DNRs are given standardized DTN names, e.g., dnr@lehigh.edu, where lehigh.edu is the group's name. The DNRs form an overlay network above the DTN. The function of a registrar is provided by one or more (for robustness) DTN nodes. The registrar is responsible for communication with the parent group(s). It offers the mandatory service of registering the members and visitors of its group. It is responsible for ensuring the authenticity and eligibility of the nodes requesting to be registered, either as a visiting node, or as a full member node. The registrar ensures that the node identifier assigned to a requesting node is locally unique. In addition, the registrar receives registration updates from group members that are visiting elsewhere. Such updates provide information on how to reach these nodes so a DNR may update appropriate gateways with the latest node' reachability information. An example of an EDIFY DTN is shown in Figure 1.

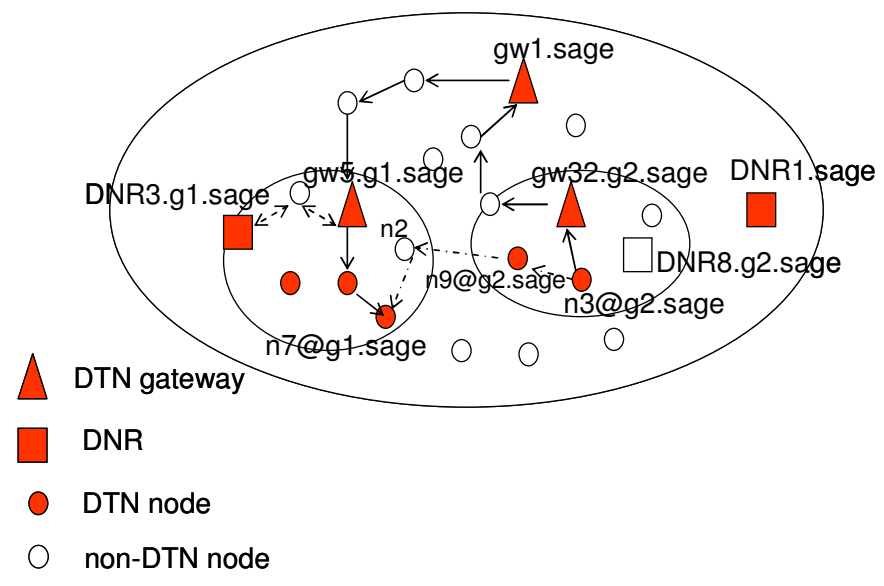

Figure 1. EDIFY DTN

In Figure 1, we show one administrative group of DTN nodes. This group is further subdivided into two subgroups. There is a DNR at the highest hierarchy with a DTN name, DNR1.sage. There is also one DNR in each subgroup with DTN names DNR8.g2.sage and DNR3.g1.sage. When node n3@g2.sage wants to send a bundle to n7@g1.sage, it checks its default forwarding policy which indicates that it 
should send any bundles destined to other subgroups of sage to gw32.g2.sage. When the bundles arrive at gw32.g2.sage, which participates in an intragroup forwarding protocol, gw32.g2.sage has a forwarding entry that indicates that any bundles destined to g1.sage should be sent to gw1.sage. At gw1.sage, there is a forwarding entry that says that bundles destined to g1.sage should be forwarded to gw5.g1.sage. Once the bundles reach gw5.g1.sage, gw5.g1.sage can seek the help of DNR3.g1.sage to resolve the DTN name n7@g1.sage to a routable address and forward the bundles to the destination node.

The above description discusses how intragroup bundle forwarding takes place. Intergroup bundle forwarding is out of the scope of this paper and will be discussed elsewhere.

\section{MOBILITY MANAGEMENT}

Since nodes move around in DTNs, we need to design a mobility management scheme to ensure that either the gateway or the DNRs know how to forward the bundles to any DTN nodes. There are three major scenarios that we need to consider:

- Individual Visiting Node Scenario. When a DTN node visits a new area, it broadcasts a DNR discovery message. Any DTN node that hears this message should respond with a unicast DNR announcement message to inform the node of the nearest DNR about which it knows. The visiting node can then register with that local DNR and possibly obtain a visiting identifier. The visiting node can also activate a forwarding feature to request that the local DNR forward a "location update" message to its home DNR. Since we are dealing with intermittently connected networks, this location update message may not arrive at the home DNR. Thus, intermediate DNRs that receive location update information will cache such information in the case that the policy of caching location update information is enabled.

- Group Visiting Scenario. When a group of nodes visits another location, instead of doing individual registrations with the local DNR, they can elect a representative, and have that representative node register with the local DNR on behalf of all of them to reduce the amount of control overhead required for location updates. This representative node will act as an impromptu gateway/DNR for the visiting group. Any group broadcast messages it receives from the home group via the local gateway will be delivered to all the nodes in the visiting group.

In the two mobility scenarios described above, after receiving the registration message, the local DNR should include the local default gateway information in its registration response message to the visiting node or the representative of the visiting group. In addition, it is assumed that the local DNR will periodically send a list of visiting nodes to the gateways within its own group and to the top-level DNR to which it belongs. This is done to ensure that if the home DNR of a visiting node issues a query about the node's latest location, the visiting top-level DNR will be able to answer that query. In addition, the visiting top-level DNR will be the proxy that sends the location update information to nearby DNRs with the intention of passing this information eventually to the home DNR if there is such an opportunity. The local DNR can set its own policy on the maximum number of DNR hops that such information will be propagated to minimize the control overhead for such location update messages.

- Mobile Network Scenario. In some scenarios, a whole network, e.g., the network hosted inside an airplane [8], may move around at a fast speed. A mobility management scheme needs to be designed to handle such mobile network scenarios as well as scenarios where a network can be partitioned into multiple groups due to geographical obstructions or enemy attacks. One approach is to have the mobile network register itself with a visiting DNR and individual nodes within this mobile network will perform registration with the DNR of the mobile network. For example, let us say bob@cse.lehigh.edu is on Plane101.SIA. Currently, Plane101.SIA is at San Francisco Airport. So, Plane101.SIA registers with the DNR of San Francisco Airport network. In addition, Plane101.SIA has pre-registered at the DNR.SIA with its flying schedule so that DNR.SIA knows which airport network to probe for the presence of Plane101.SIA at any particular time. Bob registers with Plane101.SIA and asks the DNR of Plane101.SIA to inform its home DNR (dnr@lehigh.edu) if possible of his whereabouts. Plane101.SIA can send this location update information to the DNR of the San Francisco Airport network. If there is internet connectivity between the San Francisco Airport network and Lehigh University, then Bob's home DNR can certainly get this information. Otherwise, some intermediate DNRs like the San Francisco Airport network DNR will have cached information of this location update, and will be able to answer future queries about the whereabouts of Bob.

In Figure 2, we illustrate an individual node mobility scenario. Assume that a mobile host $\mathrm{H} 1$ is visiting an area served by DNR1a at time $t_{1}$. H1 first broadcasts a DNR discovery message. After hearing a response from either a local node or DNR1a itself, $\mathrm{H} 1$ is ready to perform a registration with DNR1a. H1 turns on its home registration feature so DNR1a will be asked to act as a proxy to forward the location update information to DNR-H1, the home DNR of H1. This location update information, M3, will be 


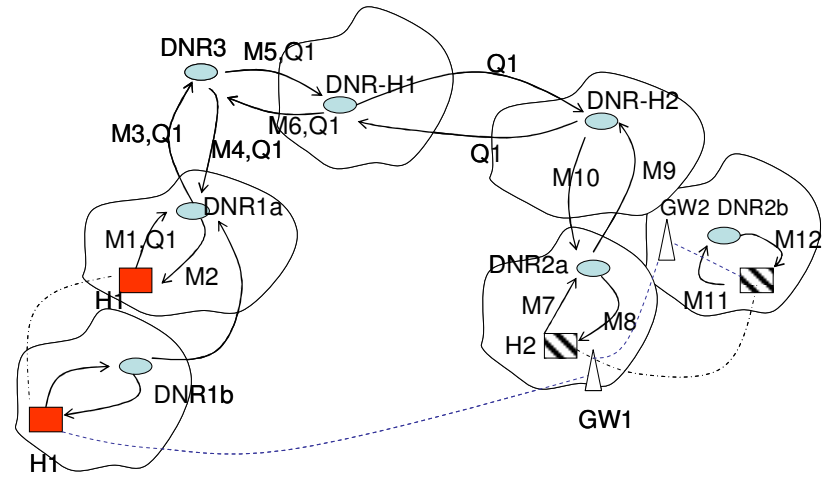

Figure 1. A Node Mobility Scenario

referred to as home location update (HLU) message. The HLU message, M3, is delivered to DNR-H1 via DNR3. Our protocol requires any intermediate DNR to acknowledge the HLU message it receives and cache the information before attempting to forward it to the final destination. So, DNR3 will respond with a HLU acknowledgment message (denoted as M4) before it tries to deliver the HLU message further to DNR-H1 (denoted by message M5). DNR-H1 will send an acknowledgment for the HLU message M5 if it receives it. If the communication link between DNR3 and DNR-H1 is not available and hence M5 cannot be delivered, then H1's location information will still be cached in DNR3 and DNR1a.

Similarly, another mobile host $\mathrm{H} 2$ which is visiting an area served by DNR2a will perform a registration after discovering DNR2a. Assume that at time $\mathrm{t}_{2}, \mathrm{H} 1$ moves to an area served by DNR1b. H1 will perform similar registration with DNR1b after discovering DNR1b. Now, H1 may not want DNR1b to send home location update message to DNR-H1 but request that DNR1b sends a location update message to its previously registered visiting DNR, DNR1a.

Now, assume that $\mathrm{H} 1$ wants to send a bundle to $\mathrm{H} 2$. $\mathrm{H} 1$ sends its bundle to the default gateway that DNR1b assigns when $\mathrm{H} 1$ performs registration. That default gateway will then query DNR1b if it does not know how to forward the bundle to H2. Alternatively, that default gateway may have information on how to send the bundle to a home gateway within the area covered by DNR-H2. When the bundle arrives at that home gateway, the home gateway queries DNR-H2 and finds out that $\mathrm{H} 2$ is now visiting at an area served by DNR2a and hence delivers the bundle to a local gateway in that area which eventually delivers the bundle to $\mathrm{H} 2$. H2 may add additional header in the bundle acknowledgement (if bundle acknowledgement feature is turned on) or a special "location update" message to H1 so that $\mathrm{H} 1$ knows its visiting identifier, and hence $\mathrm{H} 1$ can send bundles directly to $\mathrm{H} 2$ (using H2's visiting identifier) rather than via its home network.
Let us assume that $\mathrm{H} 2$ moves to an area (region) served by DNR2b while the bundles from $\mathrm{H} 1$ are being sent from its home gateway towards a local gateway in Region 2a served by DNR2a. Until H2 performs location update with DNR2b, and DNR2b shares this information with DNR2a, the local gateway in Region 2a has to store all the bundles. This local gateway also needs to query DNR2a periodically to obtain new information regarding where to forward those stored bundles destined to $\mathrm{H} 2$. Thus, we see that there are two key differences between our approach and Mobile-IP: (a) visiting DNRs cache location update information so that local gateways can query nearby DNRs for new forwarding information rather than having to rely on the Home Agent to supply such information, and, (b) local gateways need to store bundles and query local registrars for updated information to forward the stored bundles.

\section{PERFORMANCE ANALYSIS}

In this section, we perform back-of-the-envelope analysis on a simple mobility scenario to determine what fraction of the link available time a node can use for data transfer after performing location update in an environment where only opportunistic links are available. The performance metrics we consider are (a) useful utilization which is defined as the fraction of connectivity intervals that can be used for data transfer after performing location updates, (b) the latency (delay) it takes to perform location update procedure, and (c) the number of overhead messages that are generated by the mobility management scheme that we design.

The parameters in our analysis are as follows:

- $R=$ Residence time of a mobile in an area assumed to be exponentially distributed with mean $\widetilde{R}$

- $\quad l T=$ available time of a particular communication link every $\mathrm{T}$ seconds.

We assume that the communication link is opportunistic and is available for $l T$ seconds every $T$ seconds.

- $d=$ completion time for visiting DNR registration. When the link is available, it takes $d$ seconds to transfer the message across one DTN hop.

- $H=$ The number of hops before reaching a DNR. The variable, $H$, follows either (a) a truncated geometric distribution with a mean of $E[H]$ (we refer to this as Model 1). $E[H]$ is a function of two parameters, $p$, parameter of the geometric distribution, and $M$, where $M$ is the maximum number of hops in the network, or (b) an adjusted truncated geometric distribution where one can adjust the probability of the single hop scenario (this probability is referred to as $m$ ) to reflect a topology that favors a single hop scenario. (We refer to this as Model 2.) The probabilities for other hop counts 
are set to the truncated geometric distribution value but adjusted such that the sum of these values (except hop=1) sums up to $(1-m)$.

Based on our assumptions and the parameters we use, it is easy to derive that

- $E[D \mid k]$, the expected delay required to perform location update to a particular DNR given the fact that it takes $k$ hops for the location update information to traverse, $=(d+T(1-l)) * k$

- Using Model 1, the expected number of hops, $E[H]$, can be computed as

- $E[H]=\frac{1-(1-p)^{M}-p M(1-p)^{M}}{p\left(1-(1-p)^{M}\right)}$

Using Model 2, the expected number of hops, $E[H]$, can be computed as

$$
\begin{aligned}
& E[H]=m-\left(\frac{(1-m)}{\left(1-(1-p)^{M}-p\right)}\right) p \\
& +\left(\frac{(1-m)}{\left(1-(1-p)^{M}-p\right)}\right)\left(\frac{1-(1-p)^{M}-p M(1-p)^{M}}{p}\right)
\end{aligned}
$$

The expected latency of performing location update, $E[D]$ can be derived as

$$
E[D \mid H=k]=k\left(d+\frac{T}{2}(1-l)\right)
$$

After some manipulation, one can show that for Model 1 \& Model 2,

$$
E[D]=\left(d+\frac{T}{2}(1-l)\right) E[H]
$$

Assuming that the mobile host remains in its visiting area for $R$ seconds, then the useful data transfer time,

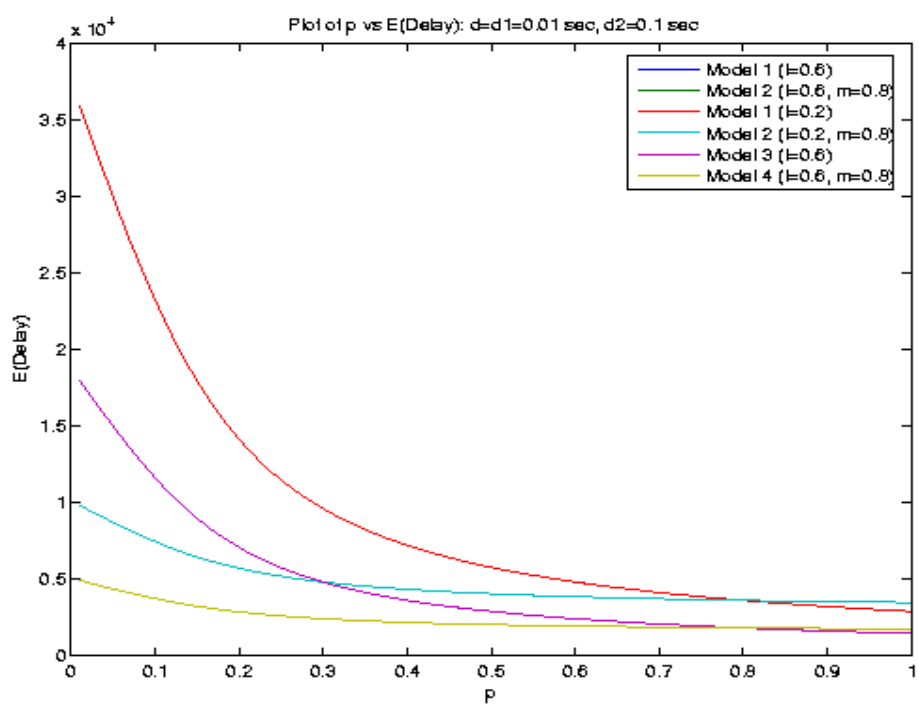

Figure 3. Expected Delay vs. $p$ (using analysis)
$U=\left\lfloor\frac{(R-D)^{+}}{T}\right\rfloor * l T$. The available data transfer time is $A=\lfloor R / T\rfloor * l T$. So, as in [10], we define the useful utilization, $u$, as $U / A$. We resort to simulations to evaluate this metric for the two models we used.

Figures $3 \& 4$ plot the expected delay versus the parameter $p$ using approximate analysis and via simulations. $M$ is set to 25 in these plots. As one can observe in Figures $3 \& 4$, the expected delay in performing the location update procedure is smaller for Model 2 since most of the scenarios in Model 2 will be single-hop scenario. Figure 4 indicates that the approximate analysis we have for expected delay matches closely with the simulation results. The useful utilization for both models when $l=0.2$ and $l=0.6$ are plotted in Figures $5 \& 6$ respectively. For these plots, we set $T=7200$ seconds ( 2 hours), and mean residence time $=3.47$ hours. For Model 1 , we set $p=0.2$ (equivalent to $E[H]=4.9$ hops) so one can observe that when $m=0.2$, the curve for Model 2 will be almost the same as the curve for Model 1 . When $m$ is larger than 0.2 (equivalent to single hop scenario being more probable than what is predicted using the truncated geometric distribution), the useful utilization improves since it takes shorter time to complete the location update procedure and hence more time can be used for the data transfer.

We also have other models where the completion time for location update may be $d 1$ seconds for the single hop scenario and $d 2$ second for the scenarios with more than 1 hop. We referred to such a model as Model 3 if we use truncated geometric distribution for the variable $H$, and Model 4 if we use the adjusted distribution as in Model 2 for the variable $H$. The derived $E[H]$ for models $3 \& 4$ are as follows:

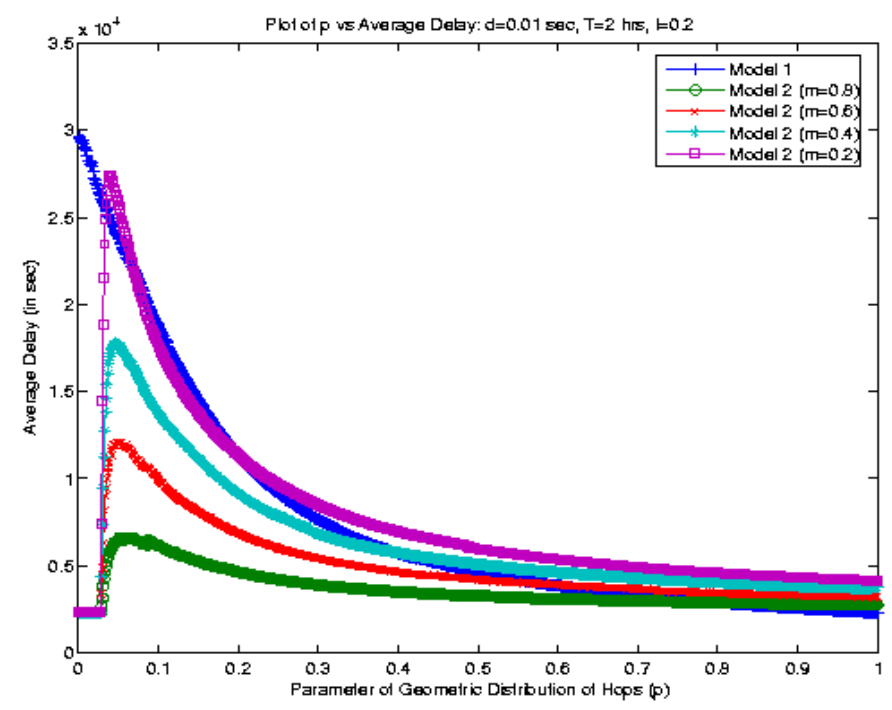

Figure 4: Expected Delay vs. $p$ with $l=0.2$ (via simulations) 


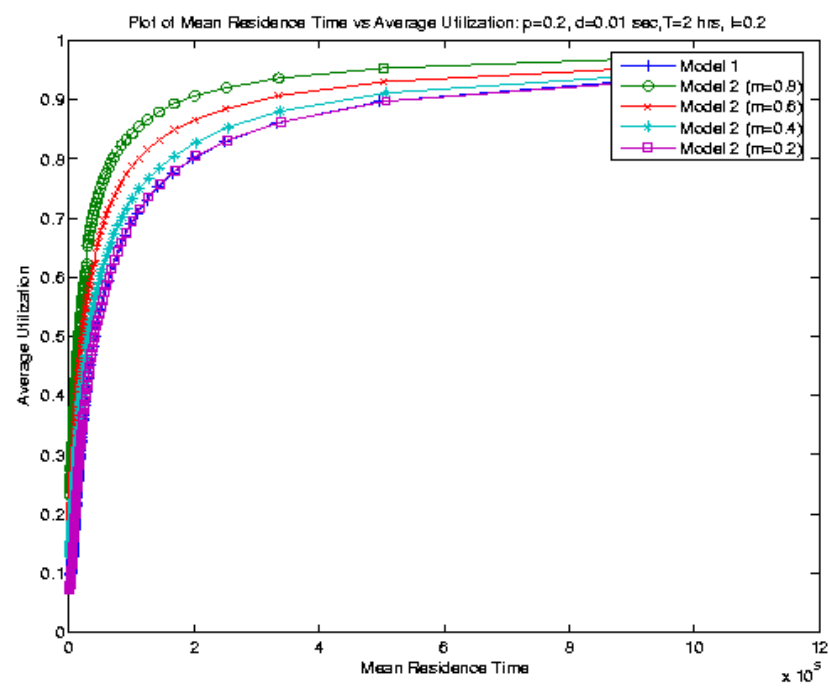

Figure 5: Average Useful Utilization versus Mean Residence Time $(l=0.2)$

For Model 3,

$$
E[D]=\left(d 2+\frac{T}{2}(1-l)\right) E[H]+\frac{p}{1-(1-p)^{M}}(d 1-d 2)
$$

For Model 4,

$$
E[D]=\left(d 2+\frac{T}{2}(1-l)\right) E[H]+m(d 1-d 2)
$$

Our further investigation indicates that the impact of different location update completion times is small since the major component of the expected latency time is the periodicity of the opportunistic link, $T$.

\section{CONCLUDING REMARKS}

In this paper, we have presented a mobility management scheme for delay and disruption tolerant networks. We have identified three major mobility scenarios that need to be addressed by the designed mobility management scheme. We then provide some back-of-the-envelope analysis to evaluate the latency involved in performing location updates in an environment where the communication links are only available for a duration of $l * T$ seconds every $T$ seconds and that the nearest DNR may be $H$ hops away from the mobile host. By putting more weight on the probability of single hop scenario, we are mimicking the near movement scenario. Our preliminary study indicates that the location update latency and useful utilization results obtained via our simple analytical model match closely with the simulation results we obtain. Such a simple model allows us to obtain useful insights into different mobility scenarios in DTNs. We are currently enhancing our simulator to simulate the three scenarios we have described. Results for the more complex scenarios will be reported in a future paper.

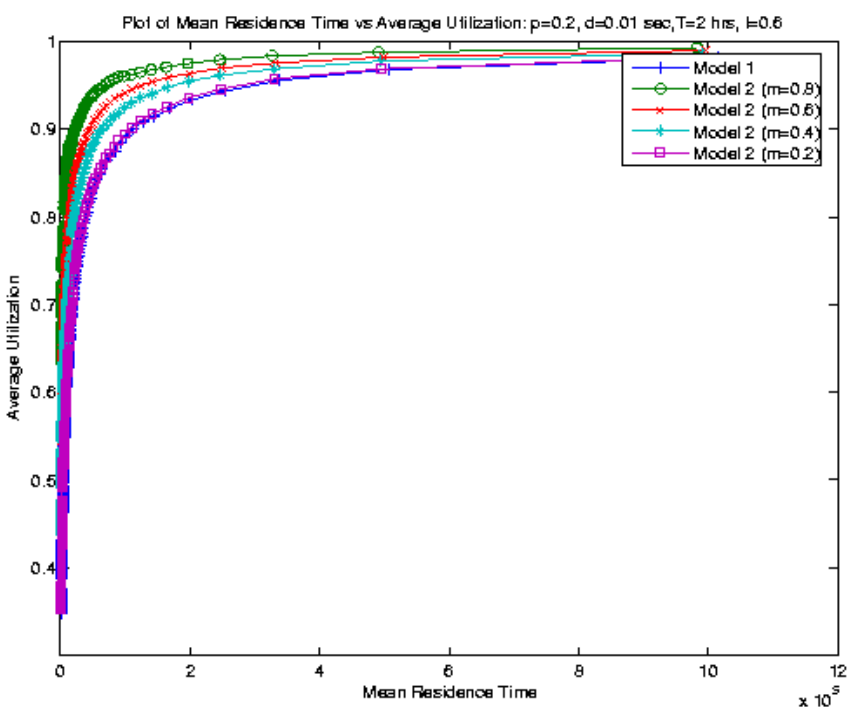

Figure 6. Average Useful Utilization vs. Mean Residence Time $(l=0.6)$

\section{REFERENCES}

[1] P. Juang, H. Oki, Y. Wang, M. Martinosi, L. Peh, and D. Rubenstein, "Energy-efficient computing for wildlife tracking: Design tradeoffs and early experiences with ZebraNet", Proc. of $10^{\text {th }}$ ACM ASPLOS, pp. 96-107, Oct. 2002.

[2] DARPA ATO, "Disruption Tolerant Networking" http://www.darpa.mil/ato/solicit/DTN.

[3] S. Burleigh, A. Hooke, L. Torgerson, K. Fall, V. Cerf, B. Durst, K. Scott, and H. Weiss, "Delay-tolerant networking: An approach to interplanetary Internet", IEEE Communications Magazine, 41(6):128-136, June 2003

[4] D. Johnson and D. Maltz, "Dynamic source routing in adhoc wireless networks", in Mobile Computing (ed. T. Imielinski and H. Korth), Kluwer Academic Publishers, Dordrecht, The Netherlands, 1996.

[5] C.E. Perkins and E.M. Royer, "Ad-hoc On Demand Distance Vector Routing", Proceedings of IEEE workshop on mobile computing systems and applications, pp. 90-100, Feb, 1999.

[6] M.C. Chuah, L. Cheng, and B.D. Davison, "Enhanced Disruption and Fault Tolerant Network Architecture for Bundle Delivery (EDIFY)”, Proc. of IEEE Globecom, Nov. 2005.

[7] K. Fall, "A delay-tolerant network architecture for challenged Internet", Proceedings of ACM SIGCOMM, pp. 27-34, 2003.

[8] The Boeing Company, "Connexion By Boeing", http://www.connexionbyboeing.com, 2005.

[9] A. Snoeren, "A Session-based Approach to Internet Mobility", PhD Thesis, MIT, http://www.cse.ucsd.edu/ snoeren, Dec 2002.

[10] A. Seth, P. Darragh, S. Liang, Y. Lin, and S. Keshav, "An architecture for Tetherless Computing", unpublished, 2005.

[11] C. Perkins (ed.), "IP mobility support for IPv4”, RFC3344, Aug, 2002

[12] A. Dutta, S. Madhani, W. Chen, O. Altintas, and H. Schulzrinne, "Fast-handoff schemes for application layer mobility management", Proceedings of PIMRC, 2004. 